\title{
Erratum: Quantitative analytical theory for disordered nodal points [Phys. Rev. B 96, 064203 (2017)]
}

\author{
Björn Sbierski, Kevin A. Madsen, Piet W. Brouwer, and Christoph Karrasch
}

(Received 9 April 2018; published 30 April 2018)

DOI: 10.1103/PhysRevB.97.139903

We point out an error in our previous article. We discuss that the functional renormalization-group (fRG) flow equations cannot be integrated in a way that is mathematically rigorous. We present results of a second-order perturbation theory calculation. In our paper, we discussed how the fRG can be used to study the effects of disorder in graphene and Weyl systems. Using a replica trick, we derived a set of fRG flow equations; their final form is given above Eq. (A9) in the Appendix of the original paper. These flow equations are correct. In our article, we solved them by simply integrating over the $\delta$-function $\delta(|i \omega|-\Lambda)$ that occurs on the right-hand side. This approach is not mathematically rigorous, which one can illustrate by considering the following, simplified case.

If in the flow equation for the self-energy $\Sigma_{\Lambda}$ we replace $A$ by the bare vertex $A_{\infty}$ and assume uncorrelated disorder (in which case $\Lambda$ serves as the UV cutoff), the self-energy takes the form $\Sigma_{\Lambda}(i \eta, k)=-i \Gamma_{\Lambda}$ with $\Gamma_{\infty}=0$. For $\hbar v \Lambda \gg \Gamma_{\Lambda}, \eta$, its flow equation is given by

$$
\partial_{\Lambda} \Gamma_{\Lambda}=-\delta(\eta-\Lambda) \frac{K}{2 \pi}\left(\Gamma_{\Lambda}+\eta\right) \ln \left(\frac{\hbar v \Lambda}{\Gamma_{\Lambda}+\eta}\right) .
$$
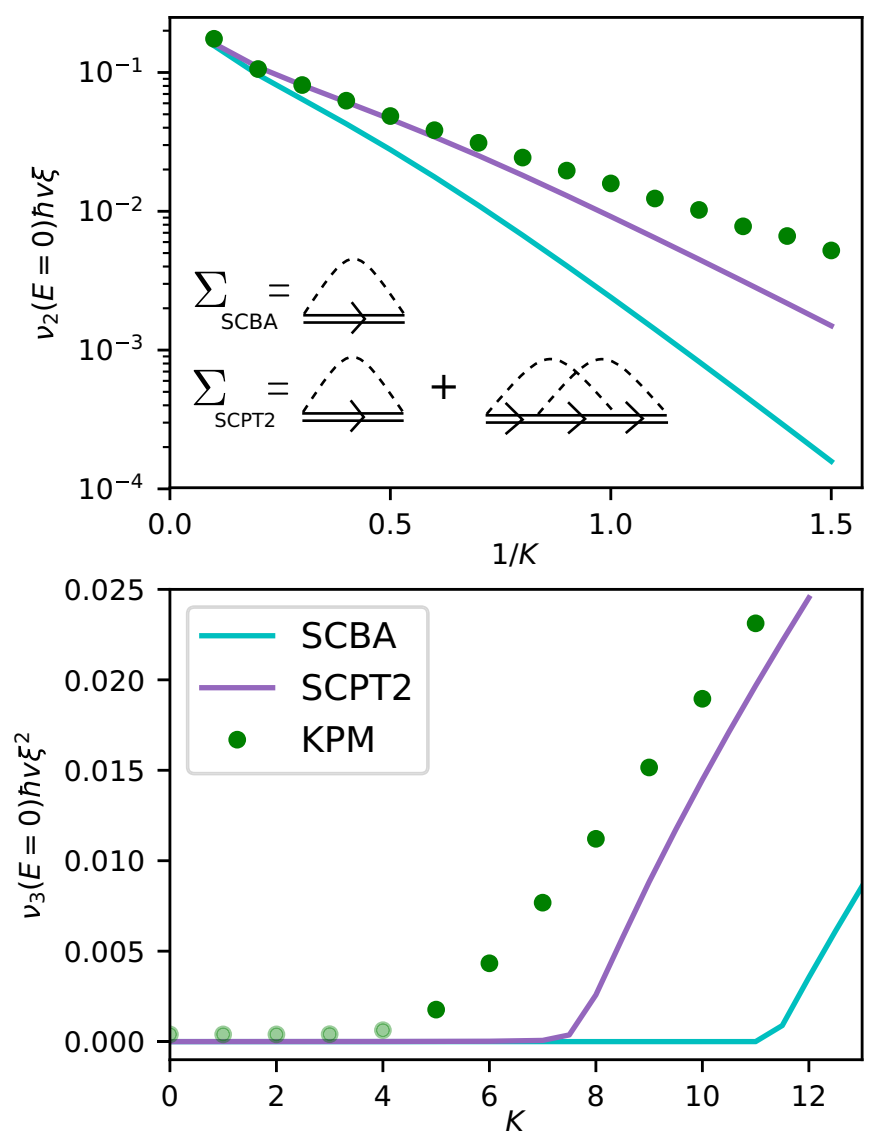

FIG. E1. Zero energy density of states of a two-dimensional disordered Dirac node $\left[v_{2}(E=0)\right.$, top] and of a three-dimensional disordered Weyl node $\left[v_{3}(E=0)\right.$, bottom] as calculated by the kernel polynomial method (KPM) versus disorder strength $K$ (the green dots). The exact KPM data are compared to the SCBA (the blue line) and self-consistent perturbation theory of second order (SCPT2, the purple line). 
This equation can be understood as an impulsive differential equation of the form $\partial_{\Lambda} f_{\Lambda}=\delta(\Lambda) g\left(f_{\Lambda}\right)$, which is analyzed in the mathematical literature. A rigorous solution to such a type of equation was constructed, e.g., in Ref. [1]. This construction, however, requires the function $g$ to be Lipschitz, which is not the case in Eq. (1) in the limit $\eta \rightarrow 0$. Thus, the theory of Ref. [1] does not apply. Despite considerable efforts, we have not succeeded in finding a mathematically rigorous solution to our flow equations [the equations above (A9) in the Appendix of the original paper, which are not Lipschitz. This problem can be corrected by using different fermionic fRG schemes, e.g., a Wick-ordered scheme with a $k$-space cutoff [2] or a two-particle-irreducible scheme [3]. Alternatively, one could employ a partially bosonized formulation where the bosonic vacuum expectation value relates to the fermionic self-energy. Results on this will be presented elsewhere.

The fact that our solution to the flow equations is flawed can also be seen by discussing the relationship with perturbation theory. In particular, the effects of static disorder on the electronic Green's function can be expressed as an infinite perturbation expansion for the electronic self-energy. The two terms of second order in disorder strength $K$ are "crossed" and "rainbow" diagrams similar as in Figs. 3(ii.a) and 3(ii.b), respectively, but with bare Green's functions (single fermion lines). Our self-consistency equation in Fig. 3 (with dressed fermion lines) was derived from the integration of (truncated) fRG flow equations. Expanding to second order in $K$ yields the aforementioned crossed and rainbow diagrams, but from diagram (i), another rainbow diagram is obtained by expanding the dressed fermion propagator. Thus, our fRG solution suffers from double counting, which should not occur for the order of the truncation.

To summarize: Eq. (A9) and the ensuing diagrammatic representation shown in Fig. 3 cannot be the proper solution to the preceding correct set of fRG flow equations since the $\delta$ function cannot be naively integrated over. We have found no way to construct a mathematically rigorous solution; corrections using different fRG schemes will be presented elsewhere. However, if we simply exclude diagram (ii.b) in Fig. 3, the resulting equation represents second-order self-consistent perturbation theory (SCPT2). In Fig. E1, we reproduce the bottom panels of Figs. 1 and 2 but replace the fRG data by the results of SCPT2 (the purple line) which improves on the first-order self-consistent perturbation theory [self-consistent Born approximation (SCBA), the blue line].

[1] M. Nedeljkov and M. Oberguggenberger, Publ. Inst. Math. 91, 125 (2012).

[2] A. Katanin, Phys. Rev. B 88, 241401 (2013).

[3] J. F. Rentrop, S. G. Jakobs, and V. Meden, J. Phys. A: Math. Theor. 48, 145002 (2015). 